\title{
CUSTO DE PRODUTOS INFANTO-JUVENIS PARA HIGIENIZAÇÃO BUCAL EM SALVADOR (BA)
}

\section{COST OF ORAL HEALTH CARE PRODUCTS FOR CHILDREN AND YOUNG PEOPLE IN SALVADOR (BA)}

\author{
Renato Magalhães Costa', Fellipe Moraes Pereira Figueiredo ${ }^{2}$, \\ Humberto Lucas Bastos de Souza ${ }^{3}$, Juliana Cristina Bastos Silva ${ }^{4}$, \\ Antônio Pitta Corrêa ${ }^{5}$, Ana Carla Robatto Nunes ${ }^{6}$
}

'Cirurgião-dentista graduado pela Escola Bahiana de Medicina e Saúde Pública, Salvador, Bahia, Brasil. renatomagalhaesc@hotmail.com ${ }^{2}$ Especialista em Odontopediatria pela Centro de Estudos Odontológicos, Salvador, Bahia, Brasil. fellipempf@gmail.com ${ }^{3}$ Cirurgião-dentista graduado pela Escola Bahiana de Medicina e Saúde Pública, Salvador, Bahia, Brasil. hlbsouza@hotmail.com ${ }^{4}$ Especialista em Odontopediatria pela Centro de Estudos Odontológicos, Salvador, Bahia, Brasil. julibastosilva@hotmail.com ${ }^{5}$ Doutor em Ciências Odontológicas. Professor na Escola Bahiana de Medicina e Saúde Pública, Salvador, Bahia, Brasil. apcorrea@hotmail.com ${ }^{6}$ Doutora em Microbiologia. Professora na Escola Bahiana de Medicina e Saúde Pública, Salvador, Bahia, Brasil. ana_carl@uol.com.br

\begin{abstract}
RESUMO I O novo paradigma de saúde adotado pela Odontologia nas últimas décadas vem priorizando a prevenção de doenças bucais. A desorganização mecânica do biofilme dentário é o padrão ouro para o estabelecimento e manutenção de um ambiente bucal saudável. Baseado nos dados recentes sobre a redução da cárie dentária no público infanto-juvenil, o presente estudo tem como objetivo estimar e analisar o custo financeiro mensal necessário para a realização da higienização bucal diária de crianças e jovens na cidade de Salvador (BA). Estudo prospectivo, transversal e de abordagem quantitativa. Em maio e junho de 2014, por meio de um formulário, foram coletadas informações básicas sobre todos os produtos infantis (escova dental, dentifrício, fio/fita dental e antisséptico bucal) encontrados em 16 supermercados de Salvador, totalizando 261 produtos. $O$ custo médio mensal em relação à escova dental foi $R \$ 1,78$, para fio/fita dental foi $R \$ 6,30$, para dentifrício foi $R \$ 4,32$ e para $\circ$ antisséptico bucal foi $\mathrm{R} \$ 36,00$. Quando associados, escova dental, dentifrício e fio/fita dental totalizaram um gasto médio mensal de $R \$ 12,40$ - valor correspondente a $1,71 \%$ do salário mínimo nacional vigente no momento da coleta de dados. Quando acrescido o antisséptico bucal aos outros três produtos, 0 valor mensal ascendeu para $R \$ 48,40(6,68 \%$ do salário mínimo vigente). Os produtos de higiene infanto-juvenis apresentam custos superiores aos produtos para adultos relatados na literatura. A utilização dos quatro produtos investigados impõe um peso significativo sobre a renda familiar, principalmente devido ao antisséptico bucal. É importante que os cirurgiões dentistas personalizem suas indicações de produtos considerando as necessidades de cada paciente e também suas condições financeiras.
\end{abstract}

\begin{abstract}
The new paradigm of health adopted by Dentistry in the last decades has been prioritizing the prevention of oral diseases. The mechanical disorganization of the dental biofilm is the gold standard for the establishment and maintenance of a healthy oral environment. Based on recent data on the reduction of dental caries in children and adolescents, the present study aims to estimate and analyze the monthly financial cost required for the daily oral hygiene of children and young people in the city of Salvador, Bahia. Prospective study, cross-sectional and with quantitative approach. In May and June 2014, a form was used to collect basic information on all children's products (toothbrush, toothpaste, dental wire and dental antiseptic) found in 16 supermarkets in Salvador, totaling 261 products. The average monthly cost with dental brush was $R \$ 1.78$, for dental wire it was $R \$ 6.30$, for dentifrice it was $R \$ 4,32$ and for the oral antiseptic it was $R \$ 36.00$. When associated, dental brush, dentifrice and dental wire totaled an average monthly cost of $R \$ 12.40$, corresponding to $1.71 \%$ of the national minimum wage at the time of data collection. When the oral antiseptic was added to the other three products, the monthly amount increased to $R \$ 48.40$ (6.68\% of the current national minimum wage). Infant and juvenile hygiene products have higher costs than the adult products reported in the literature. The use of the four products investigated imposes a significant weight on family income, mainly due to oral antiseptic. It is important that dental surgeons personalize their indications of products considering the needs of each patient and also their financial conditions.
\end{abstract}

Keywords: Oral hygiene; dental devices, oral home care; oral health.

Palavras-chave: Higiene bucal; cuidado bucal domiciliar; saúde bucal. 


\section{INTRODUÇÃO}

O mais recente levantamento epidemiológico nacional, SB Brasil 2010', apresentou algumas melhorias em relação à saúde bucal infanto-juvenil. A experiência de cárie em crianças com 5 anos foi um ceo de 2,43 - em 2003, a prevalência nessa idade era de 2,8 dentes afetados (uma redução de $13,9 \%$ ). O estudo também revelou um CPO de 2,1 na amostra com voluntários de 12 anos de idade. Esse dado representa valor $25 \%$ menor do que o encontrado em 2003 (2,8), no entanto, ainda um número superior ao $\mathrm{CPO}$ médio mundial aos 12 anos $(1,6)$.

Na Odontologia, como nas outras áreas de ciências da saúde, o paradigma da promoção da saúde ganha cada vez mais força. A prevenção de afecções bucais não somente representa melhora de qualidade de vida por sua existência, mas também por afetar a saúde sistêmica. A principal forma de instalação e manutenção de saúde bucal é obtida através da frequente desorganização e remoção do biofilme formado sobre as estruturas orais (tecidos duros e moles).

A presença de biofilme é necessária para o desenvolvimento de cárie dentária e doença periodontal, ambas doenças de complexas etiologias, sendo uma interação de estilo de vida, dieta particular e uso de fluoretos, junto com fatores do hospedeiro ${ }^{2}$.

Remoção mecânica de biofilme através da escovação e uso de fio dental tem sido universalmente aceita como padrão ouro de manutenção de saúde oral desde o início da década de $1960^{3}$.

A escovação é também um meio pelo qual foi introduzido o dentifrício, o qual é composto por substâncias químicas terapêuticas que podem melhorar a saúde bucal da população ${ }^{4}$.

Nos anos 1980, agentes quimioterápicos foram comercializados como agentes adjuntos à escovação e uso do fio ${ }^{3}$.

Existe um incentivo do mercado, não somente para a venda de produtos essenciais para higienização (escova, fio dental e creme dental), como também do colutório bucal, que para crianças tem indicação restrita. Porém, a escolha de compra de utensílios para a higienização bucal é determinada muitas vezes pelo custo financeiro dos mesmos.

O presente estudo tem como objetivo estimar e analisar o custo financeiro mensal necessário para a realização da higienização bucal diária de crianças e jovens na cidade de Salvador (BA).

\section{MATERIAIS E MÉTODOS}

Estudo prospectivo, de delineamento transversal e abordagem quantitativa. A amostra foi composta por 261 produtos infanto-juvenis para higiene bucal (escovas dentárias, dentifrícios, fios/fitas dentais e colutórios), provenientes de 16 supermercados de diferentes áreas da cidade de Salvador (BA) (amostra por conveniência). A coleta de dados ocorreu em maio e junho de 2014 e foi utilizado um formulário baseado na pesquisa feita por Carvalho et al. ${ }^{5}$.

Compuseram a amostra, produtos de estabelecimentos localizados em bairros de maior poder aquisitivo (Pituba, Barra, Rio Vermelho e Itaigara - 2 supermercados em cada bairro) e menor poder de compra (Cabula, Pau da Lima, Cajazeiras e Mussurunga - 2 supermercados em cada bairro).

Foram incluídos na amostra todos os dentifrícios, escovas dentais, fios/fitas dentais e colutórios voltados para $\circ$ público infanto-juvenil disponíveis em cada estabelecimento avaliado. Não houve seleção de produtos específicos para avaliação (determinação de produtos que seriam utilizados como referencial) devido a grande variação na presença/ausência desses itens nos locais de coletas de dados. Os critérios de não inclusão foram: produtos em promoção, combos (dois ou mais produtos vendidos em conjunto), produtos sem informações sobre o preço e produtos voltados para 
o público adulto ou sem especificação etária.

Para calcular o gasto mensal com cada produto, foi utilizada a metodologia de Carvalho et al. ${ }^{5}$, também adotada por Santos et al. ${ }^{6}$ e Costa et al. ${ }^{7}$.

Para estimar o custo mensal de escova dentária, foi feita a soma do valor de todos os produtos avaliados e esse número foi dividido pela quantidade de escovas. Dessa média aritmética, um terço foi contabilizado como o custo médio mensal. O motivo é a recomendação de substituição das escovas dentárias a cada período de três meses, em média ${ }^{5,6,8}$.

O custo mensal com dentifrício foi estimado da seguinte forma: o preço de cada produto foi divido pelo seu conteúdo (g) para definir o valor de cada grama do produto. $O$ custo de um grama $(1 \mathrm{~g})$ de todos os dentifrícios avaliados foi somado, esse número foi dividido pela quantidade de produtos avaliados (média aritmética) e, por fim, multiplicada por 54 (número de gramas utilizados mensalmente). Carvalho et al. ${ }^{5}$ estimou $\circ$ uso de $0,6 \mathrm{mg}$ de dentifrício por escovação, totalizando 1,8 gramas por dia (três escovações diárias) e 54 gramas por mês.

O cálculo para estimar o custo mensal com fio/fita dental foi desenvolvido com a divisão do valor de cada produto por seu conteúdo $(m)$, permitindo a definição do custo de cada metro de fio/fita. $O$ valor de um metro $(1 \mathrm{~m})$ de todos os produtos avaliados foi somado e foi procedida a divisão desse resultado pela quantidade dos produtos avaliados (média aritmética). Esse valor médio final foi multiplicado por 45 (quantidade de metros necessários mensalmente: $50 \mathrm{~cm}$ de fio/fita devem ser usados a cada higienização e ela deve acontecer três vezes ao dia, totalizando 45 metros/mês de fio/fita dental).

A estimativa de custo mensal com colutórios bucal foi realizada com o preço dos produtos sendo divididos pelo seu conteúdo para determinar o valor de um mililitro $(1 \mathrm{ml})$ de colutório. Os valores de $1 \mathrm{ml}$ de todos os produtos foram somados e foi calculada a média aritmética, definindo-se o valor médio de $1 \mathrm{ml}$ de colutório. Esse número foi multiplicado por 900. Os fabricantes preconizam que $10 \mathrm{ml}$ sejam utilizados a cada bochecho e que ele seja feito três vezes ao dia, o que resulta em $900 \mathrm{ml}$ por mês.

gasto mínimo mensal foi calculado através do valor do produto mais barato encontrado e o gasto máximo através do produto mais oneroso.

Os dados foram tabulados com o programa Microsoft Office Excel 2007 e apresentados por meio de estatística descritiva simples.

\section{RESULTADOS}

A tabela 1 apresenta a disponibilidade de produtos infanto-juvenis para higienização bucal nos estabelecimentos avaliados em Salvador (BA), em 2014.

Tabela 1. Disponibilidade de escova dental, fio/fita dental, dentifrício e antisséptico bucal nos supermercados avaliados no município de Salvador, em 2014.

\begin{tabular}{lcc}
\hline Produtos & $\mathbf{n}$ & $\%$ \\
\hline Escova dental & 96 & 36,78 \\
Fio/fita dental & 66 & 25,29 \\
Dentifrício & 72 & 27,59 \\
Colutório & 27 & 10,34 \\
\hline Total & $\mathbf{2 6 1}$ & $\mathbf{1 0 0}$ \\
\hline
\end{tabular}


Tabela 2. Gasto mensal individual com produtos infanto-juvenis para higiene bucal em Salvador (BA), 2014.

\begin{tabular}{lccc}
\hline Produto & $\begin{array}{c}\text { Gasto } \\
\text { Mínimo } \\
(\mathbf{R} \$)\end{array}$ & $\begin{array}{c}\text { Gasto } \\
\text { Médio } \\
(\mathbf{R} \$)\end{array}$ & $\begin{array}{c}\text { Gasto } \\
\text { Máximo } \\
(\mathbf{R} \$)\end{array}$ \\
\hline & & & \\
Escova dental & 0,49 & 1,78 & 9,96 \\
Fio/fita dental & 1,35 & 6,30 & 20,25 \\
Dentifrício & 2,16 & 4,32 & 14,04 \\
Colutório & 18,00 & 36,00 & 63,00 \\
\hline
\end{tabular}

Na tabela 3, o gasto médio mensal e sua porcentagem em relação ao salário mínimo nacional vigente em 2014 de diferentes alternativas de associações de produtos para higienização bucal.

Tabela 3. Alternativas de associação dos produtos recomendados para higiene bucal e gasto médio mensal por pessoa em Salvador (BA), 2014.

\begin{tabular}{lcc}
\hline Alternativas de associação & $\begin{array}{c}\text { Gasto } \\
\text { Médio } \\
\text { mensal } \\
(R \$)\end{array}$ & $\begin{array}{c}\text { Porcentagem } \\
\text { em relação ao } \\
\text { salário mínimo } \\
\text { vigente* }\end{array}$ \\
\hline Escova dental + Dentifrício & 6,10 & 0,84 \\
Escova dental + Fio/Fita dental & 8,08 & 1,11 \\
Escova dental + Dentifrício + Fio/Fita dental & 12,4 & 1,71 \\
Escova dental + Dentifrício + Fio/Fita dental + colutório & 48,4 & 6,68 \\
\hline
\end{tabular}

\section{DISCUSSÃO}

As medidas de higiene bucal incluem assistentes mecânicos (escovas de dentes, fio dental, limpadores interdentais, gomas de mascar) e agentes quimioterápicos (bochechos, dentífricos). O benefício derivado de higiene oral depende da destreza manual, estilo de vida, motivação e condição bucal do indivíduo?.

Sobre a disponibilidade de produtos, dos 261 avaliados, 162 (62\%) eram instrumentos mecânicos de remoção e desorganização de biofilme (escova dental e fio/fita dental). Esse resultado foi diferente do verificado por Costa et al. ${ }^{7}$, no qual os produtos de métodos químicos foram mais prevalentes $(602$ produtos - 73,23\% da amostra). Esses autores avaliaram o custo de produtos de higiene bucal em seis cidades do Estado da Bahia: Salvador (2013), Alagoinhas (2014), Brumado (2014), Jacobina (2014), Juazeiro (2014) e Senhor do Bonfim (2014). A amostra foi composta de 882 produtos não infantis (escova dental, dentifrício, fio/ fita dental e antisséptico bucal), provenientes de 32 supermercados e a metodologia de Carvalho et al. ${ }^{5}$ foi utilizada para estimar o gasto mensal com esses produtos.

O gasto médio com escova dental em Salvador foi de $R \$ 1,78$ reais, valor inferior ao $R \$ 2,71$ verificado em 2013 na mesma cidade com produtos não-infantis ${ }^{7}$. 
Escovamos os dentes por muitas razões: para sentir refrescância e segurança, para ter um belo sorriso, para evitar o mau hálito e para evitar doenças. A seleção de escova dental é, em grande parte, uma questão de preferência pessoal, acessibilidade, disponibilidade e recomendação profissional. Pode prevenir a periodontite e, certamente, previne a cárie dentária quando realizada em conjunto com creme dental fluoretado ${ }^{2}$.

Para a higienização bucal mensal com uso de dentifrício, seria necessário gastar $\mathrm{R} \$ 4,32$, valor superior ao estimado para produtos não-infantis por Costa et al.7: R\$ 2,70. Na amostra dessa atual pesquisa, não houve diferenciação entre dentifrícios fluoretados e não fluoretados. O motivo disso foi a grande variedade de dentifrícios, inclusive diferentes concentrações de fluoretos em produtos para a mesma faixa etária $(500$ ppm, 750 ppm, 1100 ppm, 1450 ppm), além do uso de diferentes substâncias para fluoretação.

Costa et al. $^{10}$ avaliaram as concentrações de fluoretos nos dentifrícios infantis disponíveis no mercado brasileiro. A busca pelos produtos foi feita através de sites de busca usando os descritores "dentifrício infantil", "creme dental infantil" e "creme dental para crianças". O refinamento das informações foi obtido através dos dados disponíveis nos sites oficiais das empresas fabricantes. Foram encontradas 28 opções de dentifrícios infantis, de 12 empresas fabricantes. Quanto a concentração de fluoretos, 11 (39,2\%) dentifrícios não continham flúor na sua formulação, $4(14,2 \%)$ apresentavam 500 ppm de fúor, 4 (14,2\%) tinham 750 ppm de flúor, 4 (14,2\%) possuíam a concentração 1100 pppm, 1 (3,5\%) apresentava a concentração de 1450 ppm e 4 (14,2\%) não traziam informações sobre a concentração de fluoretos.

Um ponto importante a ressaltar é que a metodologia de Carvalho et al. ${ }^{5}$ não foi desenvolvida para produtos infantis. Atualmente, a Academia Americana de Odontopediatria" recomenda o uso de $0,25 \mathrm{mg}$ de dentifrício fluoretado em crianças entre 3 anos e 6 anos (e 0,1 mg nos menores que essa idade). $O$ Ministério da Saúde ${ }^{12}$ preconiza que toda a população, em especial crianças menores de nove anos, deve usar dentifrícios fluoretados em pequenas quantidades (cerca de 0,3 gramas, equivalente a um grão de arroz) - dentifrícios com baixa concentração de fluoretos ou não fluoretados não são recomendados por esse órgão federal.

Dessa forma o mais correto seria afirmar que o custo mínimo, médio e máximo com dentifrícios infantojuvenis em Salvador seria de: $\mathrm{R} \$ 1,08, \mathrm{R} \$ 2,16$ e 7,02 , respectivamente - metade do valor estimado usando a metodologia de Carvalho et al. ${ }^{5}$.

O gasto médio mensal com fio/fita dental foi de $\mathrm{R} \$ 6,30$, valor também superior ao encontrado em produtos não-infantis em Salvador por Costa et al. ${ }^{7}$.

Em uma revisão sistemática, os autores examinaram os efeitos do uso do fio/fita dental (realizado pelo paciente ou por um profissional, supervisionado ou não supervisionado) em adição à escovação no manejo de doença periodontal e cárie dentária em participantes dentados de idade igual ou superior a 16 anos, em comparação com a escovação sozinha. Os resultados de 12 ensaios clínicos controlados e randomizados mostraram que pessoas que fazem uso frequente da escovação e fio dental têm menor sangramento gengival comparado com indivíduos que realizavam somente escovação. Os autores não foram capazes de determinar conclusões definitivas sobre os benefícios do uso do fio dental na redução do risco de cárie dentária, porque nenhum dos estudos incluídos havia focado nessa questão ${ }^{13}$.

Do ponto de vista terapêutico, o benefício mais óbvio do uso de enxaguantes bucais é a potencial redução de biofilme ${ }^{14}$. Os enxaguatórios bucais, mais precisamente os que possuem antissépticos na formulação, frequentemente indicados no controle químico da placa bacteriana, podem ser prescritos em situações excepcionais, entre as quais os processos inflamatórios e infecciosos instalados na região bucofaríngea com vistas à reabilitação da saúde bucal ${ }^{15}$.

Nos produtos para adultos, os colutórios são, na imensa maioria, produtos com antissépticos como principal produto componente. Essa situação é diferente nos produtos infanto-juvenis porque grande parte dos produtos para esse público é de soluções fluoretadas.

Quando indicados pelo cirurgião dentista, a 
recomendação do uso desses produtos é iniciar a partir de seis anos de idade, pois nesta fase as crianças já sabem bochechar sem deglutir a solução ${ }^{16}$.

O custo mensal estimado em Salvador com colutórios bucais correspondeu a $\mathrm{R} \$ 36$, enquanto que Costa et al. ${ }^{7}$ estimaram RS 28,50 para antissépticos bucais na mesma cidade. Os colutórios apresentaram valores superiores aos dos antissépticos, mas, além disso, foram comuns as apresentações comerciais com menores quantidades de conteúdo, o que tornava ainda mais oneroso o seu custo mensal. Por exemplo, caso seja selecionado um produto com $250 \mathrm{ml}$, será necessário o uso de quase 4 frascos completos para alcançar os $900 \mathrm{ml}$ usados por mês - por isso o custo máximo com esse tipo de produto alcançou RS 63.

A escova dental foi o único dispositivo de higiene infanto-juvenil que teve um menor custo ( $R$ \$ 1,78 $x R \$ 2,71)$ quando comparado aos resultados de produtos não-infantis verificados por Costa et al.?, também em Salvador. Esse maior custo já era previsto porque produtos infanto-juvenis frequentemente trazem em sua embalagem personagens queridos pelo público infantil. Essa abordagem ajuda a atrair a criança, estimulando a higienização, mas promove um aumento do valor dos produtos porque é necessário o pagamento do licenciamento dos personagens estampados.

A empresa fabricante Boni Brasil apresenta dois dentifrícios não fluoretados: Bony Baby Gel Dental e Bony Baby Gel Dental Disney. O primeiro estampa em sua embalagem uma imagem de um bebê e 0 outro produto apresenta $\circ$ personagem Ursinho Pooh. Em média, o dentifrício com o personagem da Disney apresenta um custo de $150 \%$ do valor do Bony Baby Gel Dental.

Santos et al. ${ }^{6}$ verificaram, em supermercados do município de Cascavel (PR) nos anos de 2004, 2005 e 2006, o custo dos produtos de higiene bucal para adultos (escova dental, dentifrícios e fio/fita dental).

Em 2006, o gasto mensal com escova dental foi de $R \$ 1,55$, com dentifrício foi $R \$ 2,16$ e com fio/ fita dental foi $R \$ 4,95$. Numericamente, todos três produtos avaliados apresentaram menor custo em Cascavel do que o que foi verificado com produtos infanto-juvenis em Salvador nessa pesquisa, mas a utilização desses três produtos somava custo mensal correspondente a $2,36 \%$ do salário mínimo nacional vigente $(R \$ 8,66)$ contra $1,71 \%$ na capital soteropolitana (R\$12,40). Portanto, apesar de possuírem valores numericamente inferiores, os produtos em Cascavel apresentavam maior custo relativo porque a população tinha um menor poder de compra - em 2006, o salário mínimo nacional era $R \$ 350,00$, e em 2014 era de $R \$ 724,00$.

Em 1986, no primeiro estudo de custo de produtos para higiene bucal realizado em Salvador (BA), Carvalho et al. ${ }^{5}$ estimaram o custo de escova dental, dentifrício, fio/fita dental e antisséptico bucal nos meses de maio e junho. A associação escova dental, dentifrício e fio/fita dental (a mais frequentemente recomendada pelos cirurgiões dentistas aos seus pacientes) custava $5,15 \%$ do salário mínimo nacional vigente em 1986 ( $C z \$ 804,00)$, número três vezes maior que o encontrado em 2014 (1,33\%) e superior ao dobro verificado em Cascavel $^{6}$, no ano de 2006 $(2,36 \%)$. O antisséptico também era o produto mais oneroso em 1986 e se fosse adicionado aos três produtos já citados, o custo mensal era elevado à $15,30 \%$ do salário mínimo nacional vigente (contra $6,68 \%$ em 2013/14 - média aritmética das seis cidades avaliadas). Esse alto custo financeiro estava relacionado à grande taxa de inflação nacional, fator que limitava o poder de compra da população na década de 1980, e que no momento da realização desse estudo (2014) apresentava (comparativamente) uma drástica redução de valores.

\section{CONCLUSÃO}

O custo médio mensal com escova dental, fio/ fita dental, dentifrício e colutório bucal foi, respectivamente, $R \$ 1,78, R \$ 6,30, R \$ 4,32$ e $R \$$ 36. O gasto médio mensal com higienização ao utilizar creme dental, dentifrício, fio/fita dentária e colutório foi de $\mathrm{R} \$ 48,40$, representando um gasto percentual de $6,68 \%$ do salário mínimo nacional vigente em 2014 .

Os produtos infantis apresentam valores elevados 
quando comparados aos produtos para adultos, exceto as escovas dentais. Isso pode ser justificado pelos produtos infantis apresentarem incluídos em seus preços finais os valores pagos para as empresas que detém a propriedade e o direito de uso de imagem dos personagens que estampam as mercadorias.

A utilização dos quatro produtos investigados infligiria um peso significativo sobre a renda familiar, principalmente devido ao antisséptico bucal. É importante que os cirurgiões dentistas personalizem suas indicações de produtos considerando as necessidades de cada paciente e também suas condições financeiras.

\section{CONFLITOS DE INTERESSES}

Nenhum conflito financeiro, legal ou político envolvendo terceiros (governo, empresas e fundações privadas, etc.) foi declarado para nenhum aspecto do trabalho submetido (incluindo mas não limitandose a subvenções e financiamentos, conselho consultivo, desenho de estudo, preparação de manuscrito, análise estatística, etc).

\section{REFERÊNCIAS}

1. Brasil. Ministério da Saúde. SB Brasil 2010. Pesquisa Nacional de Saúde Bucal - Principais resultados. Brasília. DF. 2012.

2. Yaacob $M$, Worthington HV, Deacon SA, Deery C, Walmsley $A D$, Robinson $P G$ et al. Powered versus manual toothbrushing for oral health. Cochrane Database Syst Rev. 2014;(6):CD002281. doi: $10.1002 / 14651858 . C D 002281$.pub3

3. DePaola LG, Spolarich AE. Safety and Efficacy of Antimicrobial Mouthrinses in Clinical Practice. The Journal of Dental Hygiene. 2007; Special supplement to Access Magazine:15-25.

4. Magalhães AC, Moron BM, Comar LP, Buzalaf MAR. Uso racional dos dentifrícios. RGO. 2011 ;59(4):615-25.

5. Carvalho AA, Correa AP, Amberger MI, Pinto MLMC. O Custo Social da Higiene Oral na Cidade de Salvador. Rev Fac Odontol Univ Fed Bahia. 1987;7(1):75-85.

6. Santos LF, Hirata E, Mialhe FL, Silva DD, Silva RP. Custo da Higienização Bucal no Município de Cascavel - PR. RFO. 2008;13(2):12-6.
7. Costa RM, Silva JCB, Brito AA, Vieira RS, Corrêa AP. O custo da higiene bucal na Bahia: capital e interior. In: Anais XVII ClOBA; 2014 ; Salvador (BA); Salvador; 2014. p. 99.

8. Feitosa NB, Martins CC, Chalub LLF, Vale MPP, Paiva SM. Avaliação da Apresentação Comercial de Escovas Dentais Disponíveis no Brasil. Rev Odonto Ciênc. 2008;23(1):77-81.

9. Choo A, Delac DM, Messer LB. Oral hygiene measures and promotion: review and considerations. Aust Dent J. $2001 ; 46:(3): 166-73$.

10. Costa RM, Caires AS, Nunes ACR, Alves-Neto AA. Concentrações de fluoretos em dentifrícios infantis. In: Anais XVII CIOBA; 2014; Salvador (BA). Salvador; 2014. p. 112.

11. American Academy of Pediatric Dentistry. Policy on Early Childhood Caries (ECC): Classifications, Consequences, and Preventive Strategies. Reference Manual. 2014/15;3(6):50-2.

12. Brasil. Ministério da Saúde. Secretaria de Atenção à Saúde-Secretaria de Atenção Básica, editor. Guia de recomendações para o uso de fluoretos no Brasil. Brasília - DF. 2009.

13. Sambunjak D, Nickerson JW, Poklepovic T, Johnson TM, Imai $\mathrm{P}$, Tugwell $\mathrm{P}$ et al. Flossing for the management of periodontal diseases and dental caries in adults. Cochrane Database Syst Rev. 2011 ; (12):CD008829. doi: 10.1002/14651858. CD008829.pub2

14. Morah JM. Home-use oral hygiene products: mouthrinses. Periodontology 2000. 2008;48(1):42-53. doi: $10.1111 / \mathrm{i} .1600-0757.2008 .00260 . x$

15. Araújo DB, Campos EJ, Martins GB, Araújo MTB, Assis MS, Santos TS et al. Estudo dos enxaguatórios bucais disponíveis nas drogarias, farmácias e supermercados na cidade do Salvador, BA. RBO. 2015;6(1):14-33. doi: $10.17267 / 2238-2720$ revbahianaodonto.v5i1.656

16. Macedo CR. Cuidados gerais e higiene oral para prevenção de cáries em crianças. Diagn Tratamento. 2010;15(4):191-3. 